\title{
BERLIN PAR-DELÀ LES RUPTURES. VIVRE, RACONTER ET PRODUIRE LES MATIËRES DE LA VILLE
}

\author{
Marie Hocquet, Caroline Garrido et Béatrice von Hirschhausen
}

Belin | «'Espspace géographique »

2017/2 Tome 46 | pages 158 à 173

ISSN 0046-2497

ISBN 9782410008654

Article disponible en ligne à l'adresse :

https://www.cairn.info/revue-espace-geographique-2017-2-page-158.htm

Distribution électronique Cairn.info pour Belin.

(c) Belin. Tous droits réservés pour tous pays.

La reproduction ou représentation de cet article, notamment par photocopie, n'est autorisée que dans les limites des conditions générales d'utilisation du site ou, le cas échéant, des conditions générales de la licence souscrite par votre établissement. Toute autre reproduction ou représentation, en tout ou partie, sous quelque forme et de quelque manière que ce soit, est interdite sauf accord préalable et écrit de l'éditeur, en dehors des cas prévus par la législation en vigueur en France. Il est précisé que son stockage dans une base de données est également interdit. 


\section{Berlin par-delà les ruptures. Vivre, raconter et produire les matières de la ville}

\section{Marie HOCQUET}

Université Jean Monnet, site tréfilerie Centre Max Weber, UMR 5283

6 rue Basse des rives 42023 Saint-Étienne, France hocquet.marie@gmail.com

\section{Caroline GARRIDO \\ Centre Marc Bloch, USR 3130 Friedrichstrasse 191 12161 Berlin, Allemagne garrido@cmb.hu-berlin.de}

\author{
Béatrice VON HIRSCHHAUSEN \\ CNRS-UMR 8504 Géographie-cités \\ 13 rue du Four \\ 75006 Paris, France \\ hb@cmb.hu-berlin.de
}

RÉSUMÉ .- En dépit de la réunification il y a près d'une génération, les traces du partage demeurent sensibles dans l'espace berlinois. Cet article interroge les modalités vernaculaires et quotidiennes d'actualisation de ce passé. Il analyse les processus concrets par lesquels les acteurs ordinaires effacent certaines formes urbaines héritées ou au contraire en réinstituent d'autres. Le concept de " frontière fantôme " permet ici d'articuler trois temporalités : la temporalité physique et sociale de la ville, construite et reconstruite sur elle-même, la temporalité des imaginaires continuellement réévalués qui donnent sens à l'espace urbain, la temporalité de l'expérience des habitants et passants qui pratiquent la ville.

FRONTIÈRE FANTÔME, EXPÉRIENCE, IMAGINAIRE, LIEU, MUR, TEMPORALITÉ
ABSTRACT .-Berlin beyond the breaks. Living, telling and producing the material of the city.- Despite the reunification nearly a generation ago, traces of the former division remain sensitive in Berlin. This article examines the vernacular and daily modality of the modernization of this past. It analyses the concrete processes by which ordinary actors erase certain, inherited urban forms or on the contrary restore others. The concept of "phantom borders" allows us to articulate three time frames: the physical and social temporality of the city built and rebuilt on itself; the temporalities of the imagination that are continuously reevaluated and which give meaning to urban space; the temporality of the experience of inhabitants and passerbys who use the city.

IMAGINATION, PHANTOM BORDER, EXPERIENCE, PLACE, WALL, TEMPORALITY es ruptures historiques à répé- tition qu'a connues Berlin au cours $\mathrm{du} \mathrm{xx}^{\mathrm{e}}$ siècle, ont profondément affecté la ville dans sa matérialité et ses paysages. Les urbanistes et les politiques de la ville, à chacune des périodes politiques successives, se sont employés à conformer les formes urbaines au récit historique promu par l'idéologie du moment. À chaque rupture politique majeure (1919, 1933, 1945, 1989), démolition et (re-)construction sont intervenues pour remanier les formes urbaines et leur attribuer des sens nouveaux (Riquet, 1983; Grésillon, Kohler, 2001 ; Fleury, 2009). Ces réécritures successives de la ville ne sont 
toutefois pas le seul fait des acteurs politiques et de leurs agents. Elles sont aussi le fait, plus ténu mais bien repérable, des habitants qui recomposent la ville dans leurs pratiques quotidiennes et vernaculaires (Jackson, 2003) et qui se réapproprient la ville par-delà les ruptures d'intelligibilité. Cet article ${ }^{1}$ interroge les modalités vernaculaires d'articulation du présent et du passé de la ville, les processus concrets par lesquels des habitants, des commerçants, des artistes ou de simples passants convoquent le passé dans le quotidien, l'ignorent ou le réinstituent. L'étude est conduite sur un segment emblématique de l'ancien Mur au nord des quartiers centraux, dans le sillage de la réunification. Se saisissant de ce moment particulier des décennies qui ont suivi la "Wende» ${ }^{2}$ depuis 1990, aux rives de l'ancien no man's land, dans les quartiers de Prenzlauer Berg et de Gesundbrunnen, elle s'intéresse à la manière dont des acteurs locaux effacent ou au contraire réactualisent le passé dans la matérialité urbaine.

Les processus sont saisis à travers une approche transdisciplinaire ${ }^{3}$. Nos réflexions s'appuient sur deux micromonographies de première main. L'une (encadré 1) envisage la rupture sur la longue durée - entre construction et chute du Mur - à travers les pratiques et les formes d'appropriation du territoire à l'échelle d'une rue (Gleimstrasse) qui relie deux quartiers situés de part et d'autre du Mur. La seconde étude (encadré 2) a pour objet un magasin de brocante qui a ouvert ses portes en 2005 dans le quartier de Prenzlauer Berg et qui s'est peu à peu spécialisé dans la vente d'objets issus de la culture matérielle est-allemande. Le rapport à la culture matérielle est ici envisagé dans une double dimension: d'une part, il est de l'ordre de la routine, de pratiques non questionnées, et d'autre part, il est de l'ordre de la projection, c'est-à-dire d'une mise en narration dans laquelle le passé est convoqué. Il s'agit d'interroger «[c]e qui se passe chaque jour et qui revient chaque jour, le banal, le quotidien, l'évident, le commun, l'ordinaire, l'infra-ordinaire, le bruit de fond, l'habituel» (Perec, 1989).

Le concept de "frontière fantôme» (Hirschhausen et al., 2015) est ici mobilisé pour mieux comprendre la dimension temporelle de cette production vernaculaire de la ville: comprendre comment cette production quotidienne, ordinaire, inscrite dans le temps présent, est habitée et habillée d'histoire. Berlin peut, en effet, enrichir l'inventaire de ces traces laissées dans les sociétés contemporaines par des frontières abolies. La frontière de la guerre froide y avait durement inscrit sa marque avec la construction du Mur qui continue, plus de 25 ans après sa destruction, à structurer les pratiques et les imaginaires de ses habitants comme de ses visiteurs. Cette durée des spatialités établies lors de la division Est-Ouest n'est pas du même ordre que celles observées dans d'autres études de cas proposées dans ce numéro de l'Espace géographique: à la différence des cas roumains ou ukrainiens étudiés par ailleurs et qui traitent de l'actualisation de spatialités établies il y a trois ou quatre générations, le pas de temps est ici d'une seule génération, le renouvellement des populations n'y est pas complet. La nature des phénomènes observés doit être d'abord explicitée et resituée dans l'espace de la métropole.

\section{Une "frontière fantôme "}

La longue durée de la trace du partage ne manque pas d'être intrigante. On en repère étonnamment la trace dans les cartes électorales (fig. 1): au niveau le plus fin des circonscriptions, s'y dessine l'ancienne ligne du Mur. Dans les circonscriptions du centre, les habitants ne votent pas pour la même gauche de part et d'autre de l'ancienne frontière: à l'est les votes vont majoritairement à la gauche protestataire de Die Linke, à
1. II s'agit d'une version enrichie et retravaillée d'un article publié en allemand en 2015 dans la revue Informationen zur Raumentwicklung

(Hirschhausen et al., 2015). Ce travail avait fait l'objet d'une première présentation orale en francais lors du colloque international «Berlin discontinuités: temporalités, politiques, urbanité " à l'université d'Artois en octobre 2015.

2. Mot à mot, «die Wende" signifie "le tournant». Le terme désigne en allemand le moment de la chute du régime de la République démocratique allemande (RDA) en 1989,

la réunification en 1990 et les premières années de la transformation postsocialiste.

3. Marie Hocquet est ethnologue, Caroline Garrido est historienne et Béatrice von Hirschhausen est géographe. 
Un dimanche de juin 2015 - Le Gleimtunnel est un de ces nombreux passages qui permettent de franchir le réseau dense des voies ferrées de la ville: la rue passe sous une architecture de poutres d'acier datant de l'époque Wilhelmienne. Nous sommes ici à l'extrémité nord du Mauerpark, cette large coulée verte, libérée par la destruction du Mur et réinvestie par les amateurs de karaoké, de brocante et par les touristes. Le Gleimtunnel n'est toutefois guère atteint par l'onde des foules qui, chaque week end, animent la partie sud du parc, son marché aux puces et la Bernauer Strasse. Même les habitants du quartier voisin, dans Gesundbrunnen, préfèrent le contourner ou bien ils passent au-dessus sans le voir. Au-dessus justement, les voies ferroviaires ont presque disparu, remplacées par l'herbe rase et les plantations de la coulée verte. Dans la Gleimstrasse, qui passe en dessous et relie les quartiers de Gesundbrunnen à l'Ouest et de Prenzlauer Berg à l'Est, le trafic reste modeste. Les passants sont rares à emprunter le tunnel. Plus rares encore sont ceux qui remarquent le panneau métallique «Rein, Raus» («dedans, dehors») installé à l'entrée: au gré du vent, il fait alterner le message tel une girouette. Intégré au paysage urbain, reprenant ses codes et ses attentes, tel un panneau de signalisation du quotidien d'un bleu autoroute, il est fermement vissé à chaque entrée (ou serait-ce la sortie?). La partie centrale est maintenue sur un axe, les bords sont relevés pour permettre au vent de s'agripper. C'est une installation artistique; elle date de 2010 . Elle est le fait d'arme d'un groupe d'artistes activistes*. Chacun est libre d'interpréter cette signalisation incongrue comme il le souhaite. Elle questionne et témoigne tout à la fois de la rupture** (photo 1).

Le 13 août 1961 - Un autre dimanche deux sociétés pas vraiment distinctes se faisaient face. Mises à distance par des hommes et des fils barbelés, elles se regardaient à travers le tunnel. Un tunnel miroir, bientôt un tunnel écran, coupant de la réalité de l'autre côté. Un ici et un ailleurs allaient se former et se transformer, deux destins liés et parallèles se dessinaient. Le lieu prenait un sens nouveau. En quelques jours l'autre disparaissait, avec un ici et un ailleurs repoussés au-delà du Mur, et construits désormais dans l'imaginaire. Finis terrae (photo 2).

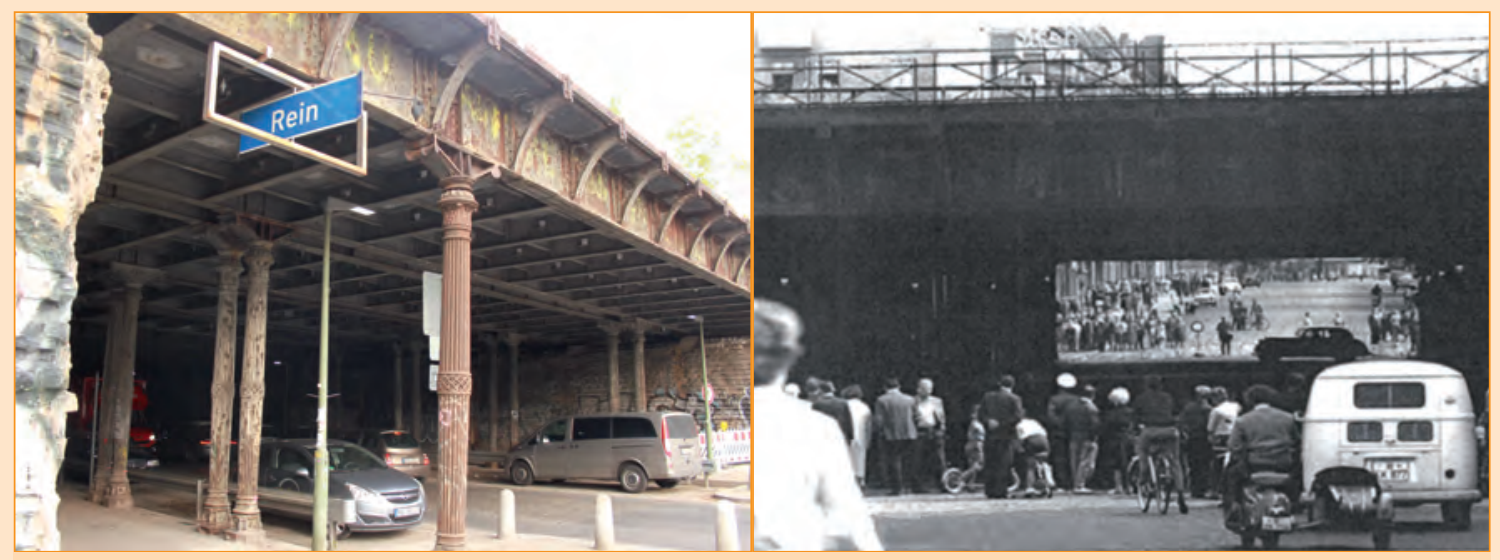

Photo $1 /$ Gleimtunnel et l'installation «Wind*»,

côté Est. Cliché de C. Garrido, avril 2017.

Photo $2 /$ Gleimtunnel, vu du côté Ouest.

Source: Landesarchiv, Berlin, août 1961.

\footnotetext{
* L'œuvre «Wind» (vent) qui a été conçue par le DNK/Filoart, un groupe d'artistes. "Unaffected by human hands, randomly driven, the weather plays with meanings. Set in an urban environment, the sign gives a confusing message which is neither a notice, a prohibition nor an advertisement. The purpose of the freedom of this arrangement is to leave the observer thoughtful, and perhaps maybe angry. Who should feel addressed? What imaginary boundary/border is the sign referring to? Who's in, who's out and who makes that decision?" (http://filoart.org/) [Indifférent et hors contrôle, allant au hasard, le vent joue avec les significations. Dans son environnement urbain, le panneau nous lance un message déconcertant, qui n'est ni une consigne, ni une interdiction ni une annonce. La liberté laissée à l'installation est là pour faire réfléchir l'observateur, peut-être même l'irriter. À qui s'adresse-t-elle? De quelle limite/frontière imaginaire est-elle le signe? Qui est dedans, qui est dehors et qui en décide?]

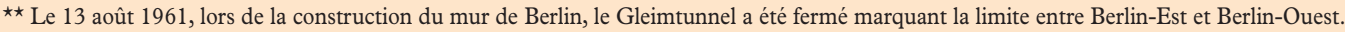


VEBorange*, février 2015 - C'est le nom d'un magasin de brocante spécialisé dans les objets du quotidien et le mobilier est-allemand qui a ouvert ses portes en 2005 au 29 de l’Oderberger Strasse, à Prenzlauer Berg. Située à quelques encablures du Gleimtunnel, l’Oderberger Strasse figure sur les itinéraires touristiques, entre la Kastanienallee et le Mauerpark ou le mémorial du Mur de la Bernauer Strasse. La rue est par ailleurs très appréciée par les habitants et les touristes en raison de l'architecture «fin de siècle» de ses immeubles, de ses nombreux bars et restaurants et de son calme piétonnier.

Le VEBorange est une véritable caverne d'Ali baba. Les objets qu'on y trouve sont issus à $80 \%$ de la production est-allemande des années 1960 , 1970 et 1980 (photos 3 et 4). Debout derrière le comptoir, Mario, le propriétaire du lieu, est en grande conversation avec des visiteurs, fumant des cigarettes autour d'une tasse de café noir. Il s'agit de collectionneurs. L'un d'une petite quarantaine, l'autre d'une dizaine d'années son aîné, parlent de manière animée. Mario se contente de leur répondre de manière un peu laconique, sans pourtant paraître ennuyé. Le plus jeune des deux hommes se saisit soudain d'une médaille exposée à proximité du comptoir: "Les Olympiades de Greifswald... J'y étais, je me souviens... ». Quelques minutes plus tard, une jeune femme d'origine espagnole fait son entrée dans le magasin suivie de deux compatriotes en visite à Berlin. Mario et la nouvelle venue se saluent chaleureusement et entament une discussion pendant que les deux excursionnistes déambulent dans le magasin, regardant et manipulant précautionneusement les objets comme s'ils se trouvaient dans un petit musée. Une femme d'environ soixante-dix ans entre à son tour dans le VEBorange: elle déballe sa précieuse marchandise: des petites assiettes en porcelaine à liserés dorés et illustrées en leurs centres de scènes pastorales. Elle certifie que ce service est de facture est-allemande ( $D a s$ ist DDR»). Mario refuse ce qui lui est proposé : ce type de vaisselle ne correspond pas aux goûts de sa clientèle.

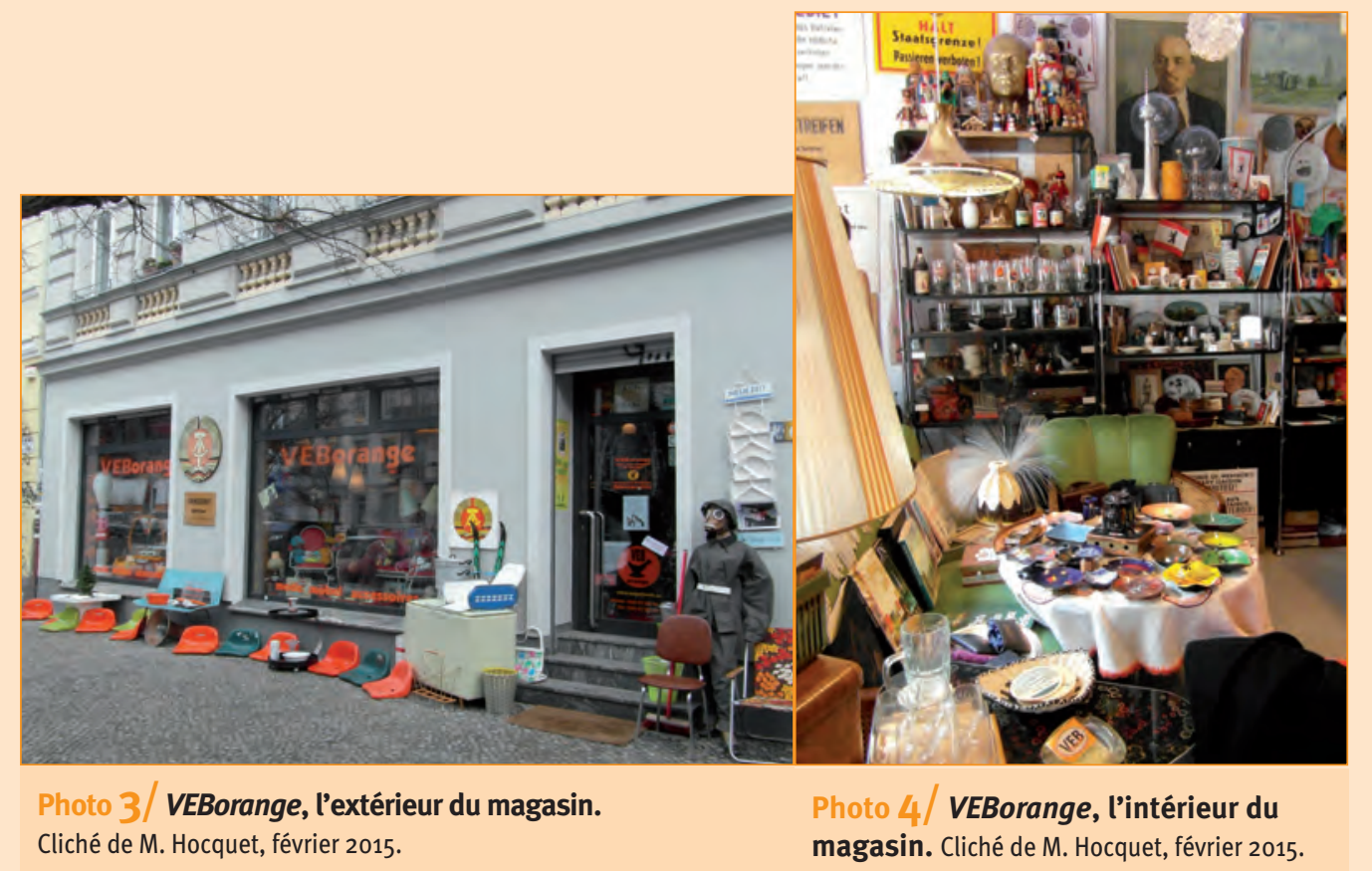

*VEBorange, le nom de cette brocante renvoie au sigle qui, du temps de la République démocratique allemande, désignait les «Volkseigene Betriebe», ces entreprises du peuple, propriétés de l'État, qui furent privatisées ou liquidées au lendemain de la réunification. 


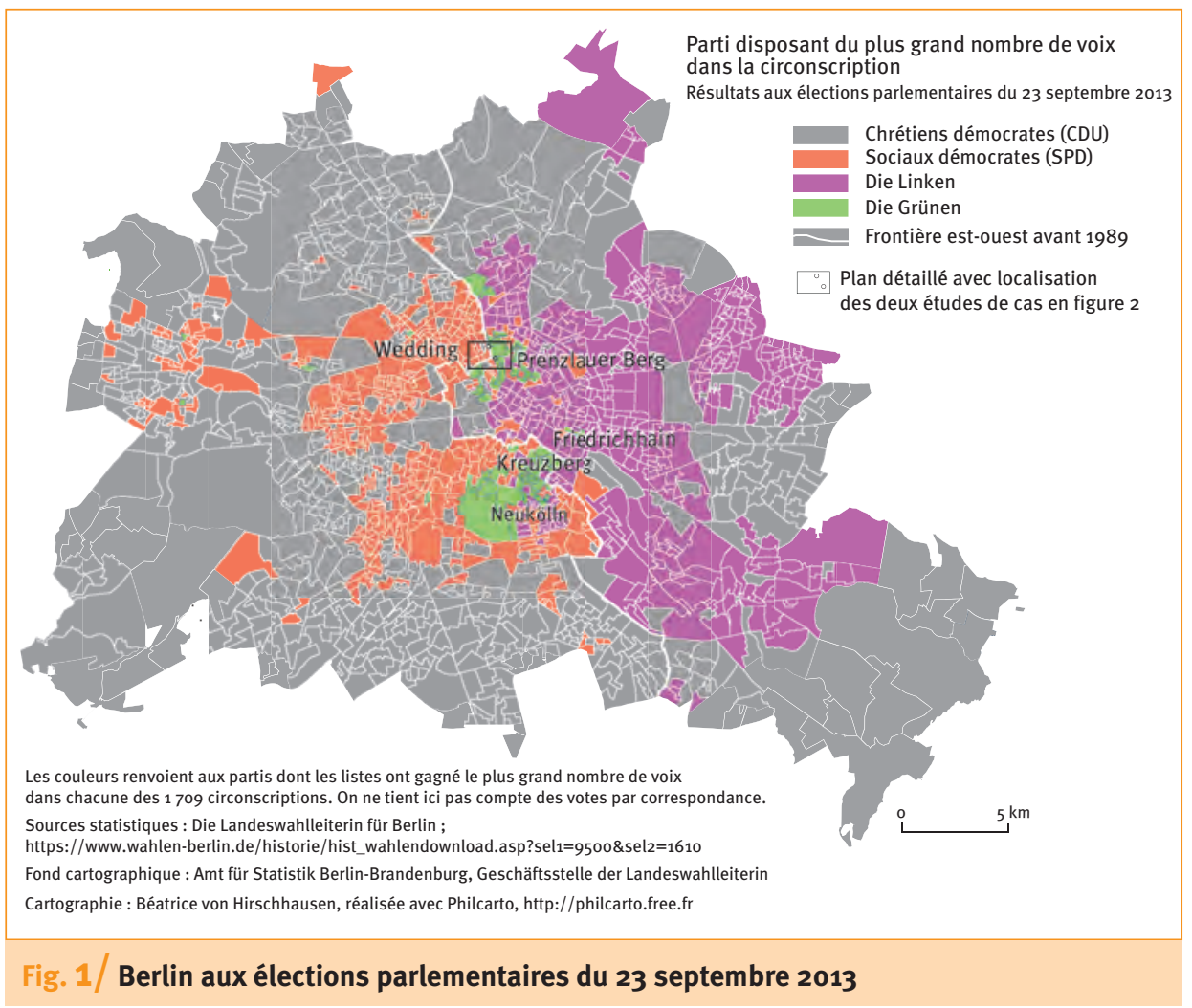

l'Ouest aux sociaux-démocrates du SPD. Le vote écologiste quant à lui, bien que présent des deux côtés, dans des aires géographiques compactes et bien individualisées, se moule aussi, à sa manière, dans les limites du partage de la ville. Au sud, il est partout majoritaire dans les circonscriptions des quartiers ouest-berlinois gentrifiés de Kreuzberg et du nord de Neukölln mais ne gagne dans aucune des circonscriptions du quartier est-berlinois voisin de Friedrichshain. Au nord, le vote vert est à l'inverse est-berlinois et correspond à la mue sociale du quartier de Prenzlauer Berg, investi par les élites créatives, mais butte vers l'ouest contre la ligne du Mur, sans pouvoir gagner aucune circonscription dans le quartier adjacent de Gesundbrunnen. La ligne de discontinuité électorale actualise ainsi le partage de la guerre froide et sépare, une génération après l'effondrement communiste, des manières différentes d'interpréter au quotidien l'expérience historique et d'envisager le futur de la cité. Le phénomène n'est pas nouveau et bien repéré par les géographes et les sociologues de la ville dès les années 1990 (Häussermann, 1997; Häussermann, Kapphan, 2000; Grésillon, 2004; Häussermann, Kapphan 2005); il nous offre des conditions quasi expérimentales pour interroger en termes comparés la production ordinaire de lieux urbains dans leur épaisseur historique. Une première analyse pourrait laisser interpréter cette géographie électorale sur le seul registre des représentations et des discours. Ce serait une affaire de mémoire vive, la mémoire "communicationnelle» de berlinois socialisés dans des univers politiques opposés, et transmise à la génération suivante. L'importance des mutations de résidence dans les quartiers centraux (Häussermann et al., 2002) laisse toutefois deviner des mécanismes plus complexes, ceux de la mémoire "culturelle» (Assmann, 1999) colportée par son 
institutionnalisation progressive dans les monuments, les musées et les commémorations, ceux aussi de l'articulation des morphologies sociales aux formes urbaines.

Les frontières fantômes se définissent à un premier degré comme les «traces laissées dans les sociétés contemporaines par des territorialités défuntes. Du fantôme, elles tiennent leur caractère imprévisible: elles peuvent apparaître sur des cartes à l'occasion d'un rendez-vous électoral [...] mais rester invisibles dans bien d'autres domaines" (Hirschhausen, 2017). À un degré plus analytique, les frontières et les géographies fantômes peuvent être saisies conjointement sur trois registres: sur le registre de l'expérience des acteurs locaux, sur le registre de leurs imaginaires et sur le registre des agencements spatiaux. L'innovation consiste ici à ne pas privilégier l'un ou l'autre de ces registres mais à explorer la manière dont ils interfèrent dans l'agir quotidien (Hirschhausen et al., 2015). Ici le recours au concept de frontière fantôme nous permet de sonder les imaginaires, les pratiques et la production sémantique et matérielle des lieux urbains par lesquelles des bribes du passé se trouvent réinstituées. La frontière fantôme se repère dans toutes sortes de signes présents dans le paysage. Les signes sont parfois ténus; en maints endroits ils disparaissent et on ne saurait dire si telle ou telle rue est de l'Est ou de l'Ouest. Mais en d'autres lieux, ils sont au contraire bien visibles, tenaces, résistant à la standardisation de la ville. De même que les fantômes ne sont pas véritablement humains, de même les frontières fantômes ne sontelles pas des frontières, au sens où elles ne séparent pas des communautés constituées et qu'elles ne font pas davantage barrière aux circulations; à la différence des frontières, les frontières fantômes ne les filtrent pas, elles ne les contrôlent pas. Elles sont des lignes de discontinuité dans l'arrangement spatial des sociétés.

Précisons ici que nous nous situons dans une conception relationnelle de l'espace. L'espace n'est pas considéré comme un support fixe ou comme un donné stable qui serait antérieur aux sociétés mais comme une dimension intrinsèque aux agencements d'objets humains ou non humains et à leurs interactions. Ce qui «fait espace» ce sont les "(dis)positions sociales et matérielles", en permanence vécues et interprétées (Löw, 2015), sans cesse réactualisées par les acteurs à travers le jeu démultiplié de leurs choix et de leurs interrelations (Werlen, 1995). Ces arrangements spatiaux se font et se défont, se tissent et se détissent sans cesse. Ils ne sauraient toutefois être compris dans le seul présent. Ils sont inscrits dans le temps historique selon différentes temporalités: temporalité de la matérialité de la ville, de son bâti, de ses trames viaires, de ses réseaux de transports tout comme de ses morphologies sociales (a), temporalités sans cesse réévaluées, des "mental maps", des imaginaires de la ville, des multiples manières dont on se la représente, dont on dessine mentalement ses contours ou ses frontières internes (b), temporalités de l'expérience historique des acteurs, de leur pratique de la ville, des lieux et des circulations (c). Le concept de frontière fantôme entend justement questionner la manière dont s'articulent ces trois temporalités : la temporalité matérielle de la ville, construite et reconstruite sur ellemême (a), la temporalité des imaginaires et des récits continuellement réévalués et ajustés qui donnent sens et organisent l'espace urbain (b), la temporalité de l'expérience des acteurs qui en deçà et au-delà des mots pratiquent la ville par leurs gestes et leurs dires quotidiens (c). Chacun des acteurs, à son niveau, pratique (c), imagine (b) et produit la ville (a). La notion de "frontière fantôme " ainsi définie nous permet de proposer une analyse originale de certains mécanismes par lesquels les traces du partage politique, puis de la réunification peuvent continuer d'être présentes dans la ville. 


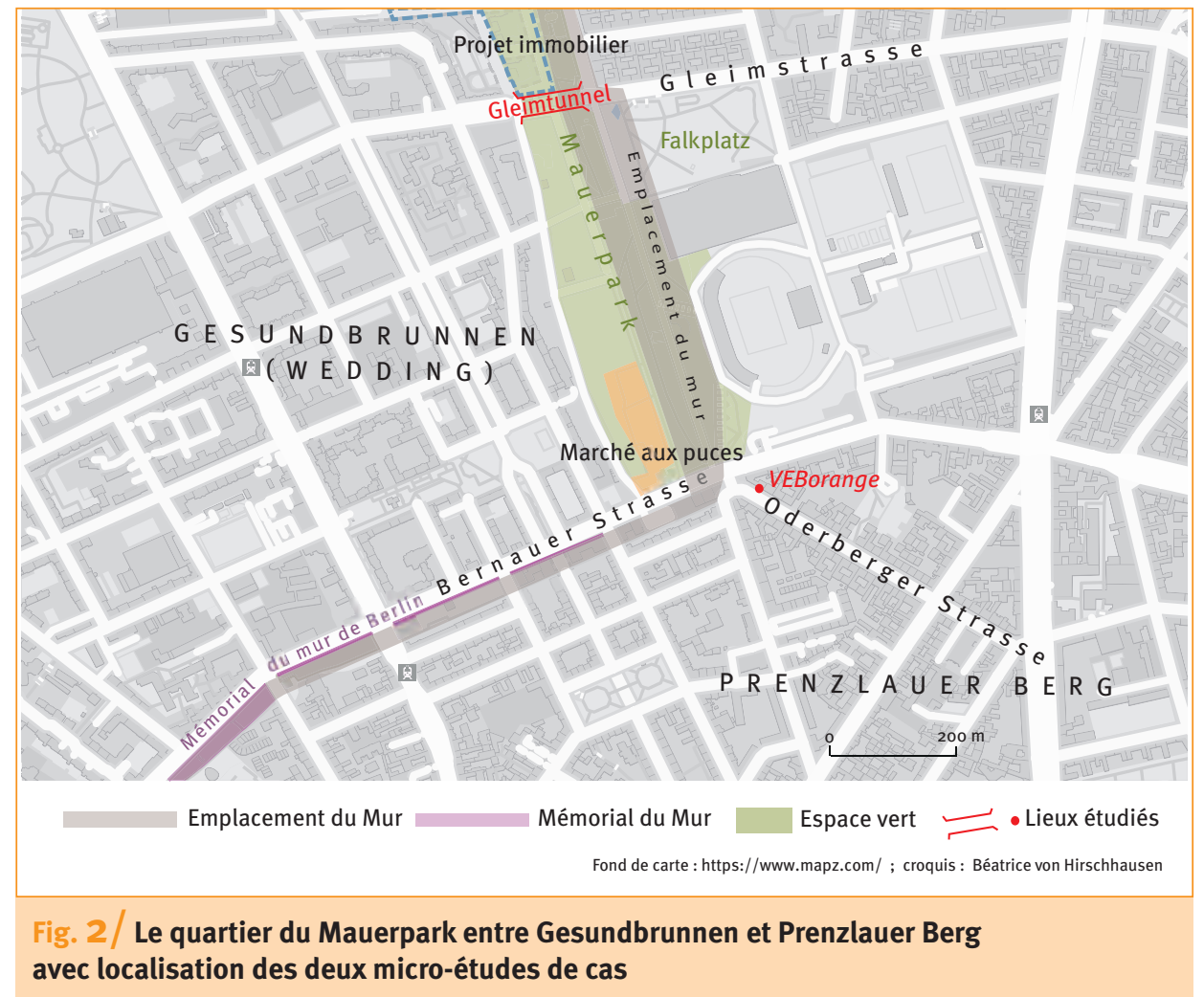

Dans les paragraphes qui suivent, nous interrogeons les matériaux décrits dans les encadrés et collectés entre Wedding (quartier de Berlin-Ouest) et Prenzlauer Berg (quartier mitoyen à l'Est) avant et après la chute du Mur. Il ne s'agit pas ici d'explorer en termes systématiques la manière dont le fantôme de l'histoire continue de coproduire l'espace de la métropole - il y faudrait un livre et un matériau empirique de grande ampleur. Plusieurs travaux qui font référence se sont d'ailleurs saisis de la question, chacun à sa manière (Terray, 1996; Grésillon, 2002; Grésillon, 2004; Reitel, 2008; Hocquet, 2011; Laporte, 2012a et b; Laporte, 2013; Fleury, 2014). Notre ambition est plus modeste. Il s'agit de mettre à jour des modalités particulières d'expression vernaculaire du fantôme à partir de matériaux collectés à une échelle microsociale.

L'étude est conduite dans une des parties de la ville qui a connu depuis la réunification, des mutations sociales et urbaines des plus profondes. La chute du Mur a rendu leur centralité à ces quartiers du nord de Mitte, situés au contact immédiat du cœur politique et culturel de la ville. Le partage de la guerre froide et ses traces y sont plus qu'ailleurs mis en scène dans des projets mémoriaux conduits par les acteurs publics et des initiatives citoyennes ou privées. La construction du mémorial du mur de Berlin le long de la Bernauer Strasse, mais aussi le succès populaire du Mauerpark établis sur l'ancien no man's land (fig. 2) en ont fait un des quartiers les plus fréquentés, par les populations jeunes comme par les touristes. La mutation n'a toutefois pas affecté ces quartiers de manière symétrique. Ils ont connu, sur leur versant est, une profonde transformation du tissu social et de la morphologie urbaine. La carte électorale en donne une première indication : la présence majoritaire du vote écologiste sur la bordure orientale 
du Mur signale l'arrivée en masse de nouvelles populations dans les quartiers gentrifiés de Prenzlauer Berg. La complexité des recompositions sociales et urbaines permet d'y explorer les relais spatiaux de la mémoire urbaine. Ici, le fantôme des spatialités de la guerre froide dans les comportements électoraux et dans les pratiques urbaines ne peut s'éclairer par le seul effet des mémoires sociales disjointes d'habitants qui continueraient de colporter deux formes différentes de représentations et de pratiques issues de deux régimes de socialisation séparés: le renouvellement des populations y est trop important. L'outillage hybride du concept de fantôme géographique, dans sa double dimension physique et symbolique voire phantasmagorique, peut nous aider à expliciter les mécanismes en jeu. Nous interrogeons ces scénettes de vie berlinoise et explorons comment s'y articulent deux à deux les trois temporalités de production de fantômes du passé dans les espaces urbains : nous recherchons comment les pratiques des acteurs sont articulées aux productions concrètes de l'espace jusque dans leur matérialité (c/a), comment ces mêmes pratiques sont également articulées aux représentations de la ville $(\mathrm{c} / \mathrm{b})$, et enfin comment ces représentations de la ville se trouvent articulées à la production de l'espace urbain (b/a).

\section{Entre pratique et production de la ville: des discontinuités rejouées}

Les acteurs pratiquent la ville au quotidien et ce faisant, façonnent sa morphologie par leurs allées et venues, leurs choix de résidence, leurs pratiques sociales, choisissant leurs espaces de prédilection, en évitant d'autres. Mais en retour, la ville organise leurs expériences sociales, les morphologies urbaines dessinent les espaces sociaux et les cadres de l'expérience. Ce mouvement dialectique identifié de longue date tant par la sociologie interactionniste que par la géographie donne une des clés des longues durées spatiales: une frontière qui a durablement structuré deux sociétés séparées peut, même une fois effacée, perdurer dans les pratiques et les morphologies sociales. Ainsi le Gleimtunnel, bien que rouvert depuis plus de 25 ans, reste-t-il peu fréquenté par les piétons des deux côtés. Les circulations des passants continuent de s'organiser de part et d'autre sans se rencontrer vraiment. Les parcours l'évitent encore aujourd'hui et les habitants des deux espaces n'échangent pas de façon régulière. Le tunnel était pourtant, avant 1961, un axe de communication très fréquenté entre les quartiers Est et Ouest. La démarcation entre les secteurs français et soviétique d'alors stimulait les échanges, les parcours se croisaient et se mêlaient. La construction du Mur n'a pas seulement établi une barrière physique quasi infranchissable, elle a réagencé les sociétés et les pratiques. Les deux quartiers ont connu dès lors des évolutions séparées. À l'Ouest, aux abords immédiats du tunnel, les rues se sont dans les années 1960 vidées de leurs commerces et de leurs habitants. Gesundbrunnen, devenu périphérie urbaine aux rives du rideau de fer, a connu une politique de logement social qui a durablement modelé l'habitat en grande partie reconstruit dans les années 1960 et 1970 . Même si le parc immobilier tend depuis vingt ans à être privatisé, cet urbanisme de reconstruction, malgré sa centralité recouvrée, ne connaît guère de processus de gentrification et garde sa population en grande partie d'origine immigrée. Celle-ci n'est pas ou peu intégrée aux puissantes dynamiques sociales et paysagères qui ont transformé l'ancien no man's land du Mur; elle ne fréquente que ponctuellement la coulée verte ${ }^{4}$ et ne s'aventure que rarement dans les quartiers embourgeoisés de l'ancien Berlin-Est. Le quartier de
4. Cette fréquentation est visible principalement le dimanche, aux beaux jours, quand les familles viennent installer leurs barbecues et leurs tables de pique-nique. 
Prenzlauer Berg sur le versant est, a en effet connu une évolution toute autre. Le Mur y était pendant la scission un repoussoir. À partir des années 1990, cet ancien quartier alors très dégradé, aimé des intellectuels ou des milieux dissidents qui n'avaient pas eu accès aux nouveaux quartiers socialistes, a connu au contraire de Gesundbrunnen, un puissant mouvement de gentrification, attirant étudiants et artistes, puis des milieux aisés ouest-allemands de moins en moins bohêmes (Häussermann, Kapphan, 2000; Häussermann et al., 2002). Ses habitants ont investi l'ancienne zone du Mur, l'ont replantée et l'on convertie en atout. Mais ils s'aventurent peu au-delà, dans le quartier Ouest de Gesundbrunnen: les quartiers reconstruits d'habitat social de l'ancien BerlinOuest sont pour eux peu attractifs. Deux urbanités sont désormais juxtaposées pour deux populations aux ressources et aux attentes très différentes. Le contraste se repère dans la trame urbaine représentée en figure 2 : à l'est perdure la maille serrée du foncier d'avant-guerre et de ses immeubles du début du $\mathrm{Xx}^{\mathrm{e}}$ siècle; elle est entamée par le très vaste aplat des installations sportives du vélodrome, au contact du Mur, mais s'est maintenu partout ailleurs, faute d'intervention urbaine dans ces quartiers délaissés par la politique est-berlinoise. À l'Ouest, on repère la maille lâche issue du volontarisme ouest-berlinois sur des ilots entièrement remembrés. Le contraste se repère aussi dans le paysage urbain, aux enseignes des commerces ou des cafés et restaurants comme dans l'architecture. Sur la carte électorale, on vote dans ces quartiers du centre berlinois, pour les sociaux démocrates à l'Ouest et pour les verts à l'Est. Les changements de population après la réunification ont ainsi fait rejouer l'effet de la frontière et les imaginaires de part et d'autre de l'ancien Mur. Inertie des espaces d'expérience et des formes matérielles de la ville se sont combinées pour "piéger" du temps en espace (Rey, 1995) et pour permettre à la discontinuité entre les deux territoires de perdurer par-delà l'effacement de la frontière et les mouvements de population. On retrouve ici les effets de l'auto-organisation systémique bien identifiés par la géographie urbaine (Lepetit, Pumain, 1993) ou dans l'analyse des systèmes productifs (Auriac, 1995).

\section{Entre pratiques et imaginaires urbains : un passé réinstauré}

Mais la présence du passé dans la ville n'est pas seulement affaire d'inertie systémique et d'auto-organisation. Elle est aussi le fait de projets actifs. L'installation artistique évoquée dans l'encadré 1, avec ses panneaux girouettes aux extrémités du Gleimtunnel, rappelle la frontière: elle en réinterprète le sens en la dédramatisant, en même temps qu'elle reconstruit la limite sur un mode subtil et rénové et qu'elle sollicite de nouveaux imaginaires urbains. D'autres projets participent plus vigoureusement encore à cette mise en scène du passé. Des acteurs économiques, associatifs ou politiques interviennent dans l'espace, mobilisent leurs expériences et leur projet pour la ville, pour réarticuler les espaces contemporains. Les alentours de l'Oderberger Strasse (encadré 2) nous en donne un exemple particulièrement intéressant. Au VEBorange, l'intervention de son patron, Mario, dans l'espace se fait au moins à deux échelles: celle du magasin lui-même et celle plus large du quartier.

Au niveau de sa brocante, il donne accès au passé est-allemand. L'établissement est un lieu de passage où se croisent plusieurs catégories de visiteurs qui, dans leur majorité, s'intéressent aux objets issus de la culture matérielle communiste: des particuliers venus proposer leurs objets à la vente, des personnes pour qui les objets vendus au VEBorange ont valeur d'objets usuels, des acheteurs occasionnels de toutes 
nationalités, des collectionneurs, des touristes et des flâneurs qui viennent observer les objets sans forcément effectuer d'achat. Le VEBorange est de facto un lieu ouvert à tous et non aux seuls initiés. Lieu de circulation de personnes et d'objets, il est tantôt qualifié de magasin, tantôt de musée par son propriétaire pour qui il ne s'agit pas seulement de donner une seconde vie aux objets, mais aussi de les "transmettre» (weitergeben). La transmission d'objets matériels se double d'une transmission immatérielle; le brocanteur devient passeur d'histoires.

Et les histoires, je les prends avec, évidemment [...]. Et les histoires, je les transmets, évidemment, ou je peux les transmettre. Par exemple, la femme qui était là - c'est juste un exemple - elle a une sœur [...] et j’ai récupéré d'elles des films en $8 \mathrm{~mm}$ des années 1950. [...] Des portraits de famille... 1954 à la plage de la baltique, j'ai ça en film $8 \mathrm{~mm}$. Un jour elles sont revenues, les mamies. [...] et je leur ai montré le film sur lequel elles étaient enfants, ici. Oui, parce qu'elles n'avaient plus le matériel [pour le visionner] et que la dernière fois qu'elles avaient vu ce film, elles étaient enfants. Et elles se tenaient là, les deux, et ça a été une expérience, non?... C'est quelque chose de très spécial. [...] et c'est aussi des choses très personnelles... Et elles racontent, les personnes âgées qui... Et c'est assez passionnant de pouvoir transmettre ces histoires.

Mario, entretien du 13 février 2015

Dans le magasin de brocante, quelque chose se produit qui va au-delà de la transaction et qui repose sur la "valeur biographique» des objets échangés (Debary, Gabel, 2010). La vente ou l'achat d'un objet à un particulier s'apparentent ainsi à des micro-événements, on y raconte des morceaux de vie, on y transmet des informations. Pour les personnes ayant vécu en RDA, ces objets suscitent des interactions qui engendrent du lien social et renforcent un sentiment d'intimité culturelle (Herzfeld, 2007). Des échanges d'informations autour des objets naît en effet un sentiment de connivence entre les initiés. Le VEBorange est donc de l'ordre du haut lieu, un lieu à travers lequel les Allemands et Berlinois de l'Est ont la possibilité de se parler d'euxmêmes, se raconter leur histoire et se rappeler leurs valeurs (Bédard, 2002, p. 234). Par ailleurs, l'usage ou le réusage de ces objets donne lieu à une réactualisation et à un partage des souvenirs qui passe par la pratique et le corps. C'est en effet par «l'apprentissage des gestes [...] permettant un engagement avec la matière» que des éléments mémoriels relatifs aux conditions de vie et aux pratiques quotidiennes $d u$ passé sont transmis (Corteel, 2015). Aussi, les objets vendus au VEBorange représentent-ils les supports d'une mémoire de la quotidienneté et d'une identité spécifique aux Allemands et Berlinois de l'Est.

Toutefois, les articles de seconde main exposés au sein du VEBorange sont également l'objet de réappropriations de la part de personnes pour qui la relation au passé communiste ne se fonde pas sur l'expérience vécue. Pour ces derniers, ces objets sont porteurs d'un témoignage sur les manières de produire en RDA et sur la culture quotidienne propre à une société qui n'est plus. Ils permettent de fait d'éprouver une réalité passée, c'est-à-dire percevoir et ressentir le passé, le mettre à l'épreuve, le tester et le mesurer, lui demander sans cesse les preuves matérielles de sa réalité révolue. Étant altérés et de ce fait chargés d'histoires qui construisent leur singularité, ces objets " permettent un passage de témoin dont l'indéfinition [...] ouvre à la redéfinition possible du 
5. L'Eberswalder Strasse est l'axe qui sépare I'Oderberger Strasse du Mauerpark et qui devient, à l'Ouest de ce dernier, la Bernauer Strasse.

6. Le mot «Kombinat» renvoie à la dénomination est-allemande désignant I'unité élémentaire de la planification étatique qui pouvait regrouper plusieurs établissements de production d'un même secteur.

7. «Le stiefelkombinat est un morceau de la liberté berlinoise - une île de diversité pour la scène autour du Berlin-vintageMauerpark ".

http://www.stiefelkombina t.de/

D'après le site, l'enseigne est désormais installée dans la Torstrasse.

8. Le terme allemand de "Kiez» désigne à Berlin des "quartiers de vie", des territoires aux limites informelles qui suffisent au quotidien de ses habitants et crée pour eux un effet de proximité dans cette agglomération particulièrement étendue. Le niveau

$d^{\prime}$ interconnaissance y est plus élevé et se noue dans les cafés, sur les aires de jeux ou dans les commerces de voisinage.

9. Nous nous basons ici sur les entretiens menés en 2014 et 2015 par Marie Hocquet dans le cadre de son enquête sur la réutilisation d'objets issus de la culture matérielle communiste et leur mise en visibilité au sein de I'espace urbain berlinois. passé» (Debary, Gabel, 2010, p. 129). Les objets vendus au VEBorange sont donc ici à envisager en tant que lieux d'une cristallisation de représentations plus ou moins stéréotypées venant nourrir un imaginaire de l'Autre.

En sa qualité d'établissement où s'effectuent des échanges (matériels et immatériels, pécuniaires et non pécuniaires) impliquant différentes catégories d'acteurs, le VEBorange est le lieu d'un brassage de visions du passé, mais également de bouts d'histoires à la fois personnelles et collectives. Les fantômes qui habitent ce lieu ne sont cependant pas assimilables à une simple résurgence du passé. Les interactions qui se jouent au VEBorange produisent en effet un arrangement (matériel, social et économique) inédit. Elles s'ancrent dans le contexte de l'après réunification et confèrent aux objets, 25 ans après la disparition de la RDA, un statut différent.

À l'échelle du quartier, le VEBorange assure également la présence du passé en l'y évoquant dans le paysage urbain pour les habitants et pour les passants. L'idée d'évocation renvoie à la visibilité de ce lieu dans l'espace berlinois. Cette visibilité résulte tout d'abord de la localisation du VEBorange. Dans le début des années 2000 il n'était pas le seul magasin à jouer sur l'évocation du passé communiste de Prenzlauer Berg. Au numéro 22 de l'Eberswalder Strasse ${ }^{5}$, se tenait par exemple le Stiefelkombinat, un magasin vintage proposant à la vente des bottes, des vêtements, des accessoires de mode et des meubles datant des années 1950 aux années $1980^{6}$. La devanture bariolée du magasin, de même que les vêtements et de nombreuses vieilles valises exposées en permanence sur le trottoir, donnaient à la rue des airs de fête. Bien que le Stiefelkombinat ait quitté l'Eberswalder Strasse, son site internet, a longtemps proclamé: "Das stiefelkominat ist ein stück berliner freiheit - Eine insel der vielfalt für die szene rund um Berlin-vintage-Mauerpark ${ }^{7}$. Ce slogan construit le «Kiez» ${ }^{8}$ autour du Mauerpark comme une scène vintage. Elle se réfère au marché aux puces du Mauerpark, mais également aux nombreuses boutiques de vintage qui se sont installées dans le quartier vers la fin des années 1990 et le début des années 2000, alors que les loyers étaient abordables et que les commerçants rivalisaient d'ingéniosité et de créativité pour composer leurs vitrines, colonisant les trottoirs d'articles d'occasion. Entre le Prenzlauer Berg gentrifié d'aujourd'hui et le Prenzlauer Berg squatté des années 1980 et 1990, il y a donc eu le Prenzlauer Berg des magasins vintages plus ou moins spécialisés dans les articles issus de la production est-allemande. Le VEBorange s'inscrit dans ce haut lieu berlinois du vintage. Le quartier connote "un certain type [...] d'atmosphère urbaine" produite par les commerces qu'on y trouve et les pratiques qu'ils suscitent (Lallement, 2005, p. 39). On se rend aux abords du Mauerpark pour chiner et négocier, observer, toucher et manipuler des objets de seconde main. Il est d'ailleurs courant d'entendre les acheteurs potentiels commenter l'aspect de leurs trouvailles et se questionner sur leur provenance (Est, Ouest?).

Si l'environnement urbain du VEBorange lui confère une part de son identité, la mise en scène du passé que la brocante propose participe en retour à sa renommée. La valeur et la spécificité du VEBorange se construit par les objets qui y sont exposés. Cette brocante est non seulement connue, mais également associée par nombre de personnes à la culture matérielle est-allemande, et à la vague d'"Ostalgie», cette nostalgie des modes de vie est-allemands qui dut sa renommée internationale à la sortie du film Goodbye Lenin!'. Ainsi l'établissement produit-il lui-même de l'espace; il crée un arrangement signifiant autour de lui et participe de la manière dont le quartier continue à être perçu et construit dans les imaginaires des habitants et des passants. 
Le quartier et le commerce se coproduisent. Le Mauerpark et ses environs, en tant que lieu de la brocante vintage et est-allemande, confèrent une plus-value sémantique au VEBorange. En retour ce dernier «signe» cette portion de Prenzlauer Berg: son geste produit un espace " habité " par le passé de la RDA que les touristes ou les nouveaux habitants " reconnaissent ", viennent chercher et contribuent à réinstaurer.

\section{Entre imaginaires urbains et production de la ville: la concurrence entre les projets}

Ces gestes producteurs de ville sont ancrés dans des représentations des espaces existants qui leur assignent valeur et sens, et dans des imaginaires tendus vers des avenirs probables ou souhaités. Ils viennent bien sûr des grands acteurs institutionnels et des investisseurs occupés dès 1990 à réunifier la ville, à lui conférer une nouvelle image et de nouvelles fonctions (Grésillon, 2003; Reitel, 2008; Laporte, 2012a et b; Laporte, 2013). Ils sont venus aussi de l'extraordinaire floraison d'initiatives artistiques venues, après la chute du Mur, de tous les horizons, s'emparer d'espaces en pleine mue et donner corps à leurs utopies, le temps d'une décennie (Grésillon, 2002). Mais les gestes producteurs de ville sont aussi vernaculaires. Les passés auxquels ils se réfèrent varient selon les acteurs. Résidents ou touristes, anciens habitants ou nouveaux venus, pour certains précaires, pour d'autres embourgeoisés, activent des narrations concurrentes de la ville, contribuent à légitimer et actualiser dans le tissu urbain, différents moments de l'histoire, différents régimes d'urbanité et de société. De ces projets entrechoqués, Berlin sort avec un fort degré d'hétérogénéité. Berlin n'est pas la ville d'une narration historique linéaire: son espace donne à voir côte à côte des projets politiques multiples et contradictoires qui ne convoquent pas les mêmes fantômes du passé. Les contrastes ne sont pas seulement Est-Ouest. Ils se jouent aussi au sein même des quartiers où se mêlent les formes urbaines comme autant de manifestes politiques.

L'aménagement de l'ancien no man's land sur les hauteurs du Gleimtunnel illustre ces débats et contrastes. Ainsi la discussion est-elle intense parmi les membres de l'association "Welt Bürger Park». Cette association lie des habitants qui pour la plupart avaient investi ce quartier de Berlin-Est situé à la lisière du Mur après sa chute. Ils ne sont pas tous Berlinois ou même Allemands ; ils ont contribué cependant à façonner le quartier tel qu'il est aujourd'hui à l'image d'un Berlin qu'ils voulaient vert et étranger à la spéculation. Leur association vise à maintenir le tracé du Mauerpark et des espaces verts qui ont réinvesti l'ancien no man's land. Ils défendent aujourd'hui, sur les hauteurs du tunnel, le long de l'ancien tracé du Mur, une ferme éducative, des espaces verts et de détente, un mur d'escalade. Dès le début des années 1990, les arbres avaient été plantés, des cohortes volontaires s'étaient succédé pour occuper l'espace autrefois parcouru par les patrouilles des gardes-frontière. L'initiative avait émergé lors des discussions d'un "Runde Tisch" ${ }^{10}$, non loin de là sur la Falkplatz. Le projet de ferme éducative avait ensuite pris de l'ampleur, impliquant les administrations (de la ville et du quartier), les écoles et les habitants, et migré sur les hauteurs du tunnel sur des terrains prêtés par la ville. Mais ce territoire est aujourd'hui convoité par un entrepreneur qui assure vouloir construire des logements sociaux. Les membres de l'association se méfient d'un projet soupçonné d'annoncer du logement social pour mieux construire du logement de luxe et s'y opposent vigoureusement. Ils cherchent à
10. Les "Runden Tische» ( "Tables rondes») désigne les réunions citoyennes qui se sont tenues en 1989 et 1990 de manière décentralisée (organisation de la vie locale par les habitants, réorganisation administrative) dans le sillage de la "Table ronde centrale» (der "zentrale runde Tisch ") qui réunit le gouvernement central et les partis d'opposition pour décider des nouvelles orientations suite au tournant de 1989. 
le bloquer. Mais si l'on interroge Wolfgang - un commerçant lié de longue date avec le quartier - sur le conflit, il éclate de rire et ironise sur les militants de «Welt Bürger Park» installés dans les appartements d'immeubles aujourd'hui complètement rénovés. Il a observé le quartier évoluer, les échafaudages se monter et se démonter les prix immobiliers s'envoler. Il tape quelques coups au mur d'une façade: ça sonne creux! Elle ressemble à de la pierre mais il n'en est rien. Alors, dit-il, on peut bien leur construire de gros immeubles en face de leurs fenêtres: eux aussi ont transformé son quartier ${ }^{11}$. Son Berlin, c'était le Berlin aux façades ridées et accidentées de Berlin-Est; ce Berlin-là s'évapore. Son Berlin est resté dans sa mémoire: il est gris. On lit aujourd'hui sur la carte des élections parlementaire de 2013 que dans ce quartier, ce Berlin-là a perdu : la gentrification est bien avancée, la circonscription est passée au parti des Verts ("die Grünen»).

Le cas du VEBorange montre également comment le registre matériel de la production urbaine s'articule aux imaginaires, aux représentations du passé et aux projets pour la cité. À l'échelle de sa brocante, Mario conteste l'ordre existant en même temps qu'il s'en arrange. La récupération d'objets usagés et leur remise sur le marché est une pratique sous-tendue pour lui par un ensemble de valeurs: la valeur d'usage d'un objet et sa longévité en opposition à la mise au rebut, la dépense inutile ou l'obsolescence programmée. Les objets est-allemands cristallisent cette prise de position.

"Je vais aussi le publier sur mon site internet, ce qu'était VEB: "Volkseigener Betrieb". [...] Beaucoup de gens ne peuvent s'imaginer que les entreprises étaient du côté du peuple [...]. L'essai [d'édifier] un État socialiste, où les... L'idée en soi, ça vaut le coup d'y penser parce que, oui, les choses qui étaient créées avec peu... avec les moyens du bord, quasiment... [...] et qui, malgré ça, étaient créées pour le long terme, enfin sans... sans n'importe quelle pièce à l'intérieur qui se retrouve fichue au bout de deux ans. Ce n'était pas la société du gaspillage (Wegwerfgesellschaft) [...] On devait toujours réutiliser les choses et le recyclage en tant que tel existait déjà en RDA. Les bouteilles et le papier utilisés étaient portés au traitement des déchets. Ça existait déjà et on a essayé de tirer le meilleur des matériaux disponibles. [...]. Et les gens... Les gens qui ont produit cela [...] c'était aussi des travailleurs qui ont reçu des lettres de la population: "Mais qu'estce que vous nous avez produit là ?" ("Was habt denn ihr für 'n Scheiß produziert?!) [...], non? Enfin, chacun pouvait se manifester et dire "Mais qu'est-ce que vous fabriquez dans vos volkseigenen Betriebe?». [...] Un peu comme si chacun pouvait participer à la production, concernant certaines choses.» Mario, entretien du 13 février 2015.

Ici le fantôme (les manières de consommer, de produire et de recycler en RDA) resurgit pour adresser une critique aux manières dominantes de produire et de consommer. Le passé se trouve mis en récit en fonction du contexte discursif présent dans lequel sauvegarde de l'environnement, recyclage et durabilité sont hautement valorisés. Ce faisant, le VEBorange est inscrit entre passé, présent et futur, en vertu d'un imaginaire où s'articulent des images, des idées et des valeurs qui ont trait à la durabilité des objets, à la participation des usagers à la production, à la consommation

11. Ces propos sont tirés d'une discussion informelle avec le commerçant en août 2014. raisonnée et au recyclage. Le VEBorange est ainsi porteur d'un récit singulier sur le passé qui, à bien des égards, contrecarre un type de discours dans lequel le territoire de la RDA est appréhendé et construit sous le vocable de la souillure (pollution des sols, de l'air, villes grises et sales, etc.; Hocquet, 2011; Herzfeld, 2007, p. 68). On voit 
bien ici comment un imaginaire du passé est mis à contribution pour concevoir et justifier un projet qui, une fois réalisé, participe à la production d'un espace et d'une urbanité connotés.

\section{Conclusion}

Les expériences de la ville ici rapportées nous offrent les exemples de différentes modalités par lesquelles Berlin se vit, se raconte et se produit au quotidien ; des modalités parmi bien d'autres, par lesquelles à Berlin, le passé est éprouvé dans la matière même de la ville. Les traces des différents passés sont tantôt là comme des résidus oubliés, tantôt comme des signes convoqués. La ville semble " habitée " par la mémoire de son histoire. C'est le fait des lieux, des paysages urbains qui donnent à sentir l'histoire au détour d'une rue; c'est aussi le fait des différents groupes sociaux qui pratiquent la ville (durablement ou de manière épisodique) et qui tous à leurs manières, redonnent vie aux fantômes d'un passé dédoublé et révolu.

L'étude croisée des deux micromonographies permet de saisir comment les matières de la ville - à travers des manières de dire, de vivre et de produire l'urbain dessinent les contours de frontières fantômes, mettant en scène, traduisant et réactualisant des discontinuités et un passé révolus. La lecture que nous proposons de ces lieux d'étude récuse l'idée d'un passé sédimenté dans l'espace en couches successives. Elle met plutôt en lumière des processus actifs, créatifs, contradictoires, mis en œuvre par différents types d'acteur pour construire "leur " projet pour la ville, en se situant dans différentes continuités historiques plus ou moins revendiquées.

Le VEBorange correspond à une production d'espace en acte qui procède d'un double mouvement de convocation du passé et de réponse commerciale à une demande actuelle. Ce magasin de brocante donne ainsi à expérimenter concrètement, dans les objets qu'il met en scène, des bribes du passé communiste qu'il a réagencées selon un dispositif qui conteste les représentations canoniques et officielles de ce passé tout en s'alignant sur une demande de consommation de ce passé.

Dans le cas du Gleimtunnel, la frontière aujourd'hui disparue suscite des imaginaires, des urbanités qui sont loin d'être univoques. À l'image du panneau métallique "Rein, Raus» ("dedans, dehors»), l'interprétation reste ouverte. Quel est le «vrai» Berlin ? Berlin doit-il être vert, orange ou gris ? Inlassablement, la ville continue de se construire " en fragment " (Şenocak, 2003), par et pour les occupants, permanents ou temporaires, volontaires ou attentistes. De cette forme d'indécision urbaine, en plein centre d'une métropole multimillionnaire, Berlin tire sa "poétique " (Sansot, 2004) et son pouvoir de fascination. Pour combien de temps encore? La valorisation immobilière et commerciale de ce capital touristique pourrait aussi signer la fin de son pluralisme. 
Assmann A. (1999). Erinnerungsräume, Formen und Wandlungen des kulturellen Gedächtnissses. Munich: Verlag C.H. Beck, $424 \mathrm{p}$.

Auriac F. (1995). "Temps et espace géographique ». In Nicolescu B., Dodille N., Duhamel C., Le Temps dans les sciences. Que fait le temps à l'affaire ? Paris : L'Harmattan, p. 85-92.

BÉDARD M. (2002). «De l'être-ensemble à l'être-au-monde: le rôle du haut-lieu ». Ethnologies, vol. 24, n 2, p. 229-241.

Corteel D. (2015). «Pratiques de récupération et transmission. Éléments de réflexion autour de la mémoire et des déchets ». Communication à la conférence internationale «Penser la mémoire entre la France et l'Allemagne: quel dialogue entre histoire et sociologie?», 28 et 29 mai, Centre Marc Bloch, Berlin.

Debary 0., Gabel P. (2010). "Seconde main et deuxième vie. Objets, souvenirs et photographies ». Mélanges de la Casa de Velázquez, nº 40-1, p. 123-142.

FLeURY A. (2009). «Berlin/Istanbul. La rue au service de l'image des métropoles». Géographie et cultures, $n^{0} 71$, p. 111-130.

Fleury A. (2014). " La mise en patrimoine des espaces publics. Regards croisés à Paris et à Berlin ». In Djament-Tran G., San Marco P. (dir.), La Métropolisation de la culture et du patrimoine. Paris: Éditions Le Manuscrit, coll. «Fronts pionniers», p. 221-240.

GrésILLon B. (2002). Berlin. Métropole culturelle. Paris: Belin, coll. « Mappemonde», 352 p.

GRÉSILLON B. (2003). «Les hauts lieux berlinois: une réappropriation problématique ». Les Temps Modernes, n 625-4, p. 119-127.

GRÉSILLON B. (2004). «Berlin, frontières anciennes, frontières actuelles». Bulletin de l'association des géographes français, vol. 81, nº 4, p. 466-475.

GrésILLon B., KoHler D. (2001). « Berlin, capitale en attente ». Hérodote, nº 101-2, p. 96-121.

Halbwachs M. (1997). La Mémoire collective. Paris : Albin Michel, coll. «Bibliothèque de l'Évolution de l'Humanité», $304 \mathrm{p}$.

Häussermann H. (1997). "Social transformation of urban space in Berlin since 1990 ». In Käelltorp O., Elander I., ERICsson O. and Franzén M. (dir.). Cities in Transformation - Transformation in Cities. Social and Symbolic Change of Urban Space. Aldershot: Avebury, p. 80-97.

Häussermann H., Holm A., Zunzer D. (2002). Stadterneuerung in der Berliner Republik. Modernisierung in Berlin-Prenzlauer Berg. Wiesbaden: Springer Fachmedien Wiesbaden GmbH, 244 p.

Häussermann H., Kapphan A. (2000). Berlin: Von der geteilten zur gespaltenen Stadt? Wiesbaden: Springer Fachmedien Wiesbaden $\mathrm{GmbH}, 292 \mathrm{p}$.

Häussermann H., Kapphan A.. (2005). « Berlin: from divided to fragmented city ». In Hamilton F.E.I., Andrews K.D., Pichler-Milanovi N. (dir.), Transformation of Cities in Central and Eastern Europe. Towards Globalization. Tokyo, New York, Paris: United Nations University Press, p. 189-222.

HiRSChHAUSEN B. von (2017). "De l'intérêt heuristique du concept de "fantôme géographique" pour penser les régionalisations culturelles ». L'Espace géographique, t. 46, nº 2, p. 106-125.

Hirschhausen B. von, Garrido C., Hocquet M. (2015). "Jenseits politischer zäsuren? Berliner Phantomgrenzen in aktuellen Pratiken und Diskursen». Informationen zur Raumentwicklung, $\mathrm{n}^{0} 5$, p. 451-460.

http://www.bbsr.bund.de/BBSR/DE/Veroeffentlichungen/IzR/2015/5/Inhalt/dl-hirschhausen-garrido-hocquet.pdf?_blob=publicationFile $\& v=2$ 
Hirschhausen B. von, Grandits H., Kraft C., Müller D., Serrier T. (2015). Phantomgrenzen im östlichen Europa, Band 1: Phantomgrenzen - Räume und Akteure in der Zeit neu denken. Göttingen: Wallstein Verlag, $224 \mathrm{p}$.

Herzfeld M. (2007). L'Intimité culturelle. Poétique sociale dans l'État nation. Québec: Les Presses de l'université Laval, coll. «Intercultures», 286 p.

HOCQUET M. (2011). Mémoire, oubli et imaginaires urbains, étude de deux hauts-lieux de la mémoire communiste à Berlin-Est: le Palais de la République et le Musée de la Stasi. Saint-Étienne: Université Jean-Monnet, thèse de doctorat de sociologie et anthropologie politique, $484 \mathrm{p}$.

JACKSON J.B. (2003). À la découverte du paysage vernaculaire. Arles, Versailles: Actes Sud, École nationale supérieure du paysage, $288 \mathrm{p}$.

LALLEMENT E. (2005). «Tati et Barbès: différence et égalité à tous les étages ». Ethnologie française, vol. $35, n^{\circ} 1$, p. 37-46.

LAPORTE A. (2012). De Bonn à Berlin. Le transfert d'une capitale (1990-2010). Toulouse: Presse universitaire du Mirail, coll. «Villes et Territoires», $266 \mathrm{p}$.

LAPORTE A. (2012) «La résilience des espaces du pouvoir et de leur structure : l'exemple de Berlin après la réunification allemande». In Djament-Tran G., ReghezzA-ZIT M. (coord.), Résiliences urbaines. Les villes face aux catastrophes. Paris: Éditions Le Manuscrit, coll. «Fronts pionniers », p. 103-133.

LAPORTE A.. (2013) « L'empreinte spatiale de l'ancienne frontière interallemande dans le Berlin d'aujourd'hui ». Belgeo, no 1. https://belgeo.revues.org/10645

LePetit B., Pumain D. (dir.)(1993). Temporalités urbaines. Paris : Anthropos, coll. «villes », 316 p.

Löw M. (2015). Sociologie de l'espace. Paris: Éditions de la Maison des sciences de l'Homme, coll. «Bibliothèque allemande », $302 \mathrm{p}$.

LöwIS S. von (2015). «Phantom borders in the political geography of East Central Europe: An introduction ». Erdkunde, vol. 69, n² 2, p. 99-106.

PéreC G. (1989). L'Infra-ordinaire. Paris : Seuil, coll. « Librairie du xxle siècle », 122 p.

Reitel B. (2008). «Pouvoir politique et production de l'espace urbain : histoire des politiques, d'aménagement urbain à Berlin ». In Vallat C. (dir.), Le Blanc A., Philifert P. (coord.), Pérennité urbaine, ou la ville par-delà ses métamorphoses. Vol. 1: Traces. Paris: L'Harmattan, p. 35-48.

REY V. (1995). "Temps piégé et espace géographique dans l'Europe de l'entre-deux ». In Nicolescu B., Dodille N., Duhamel C., Le Temps dans les sciences. Que fait le temps à l'affaire? Paris: L'Harmattan, p. 93-100.

RiQuet P. (1983). «Berlin comme volonté et comme représentation ». Hérodote, n² 28, p. 101-127.

Sansot P. (2004, $1^{\text {re }}$ éd. 1971). Poétique de la ville. Paris: Éditions Payot \& Rivages, coll. «Petite bibliothèque Payot», $626 \mathrm{p}$.

Şenocak Z. (2003). «La capitale du fragment ». Les Temps Modernes, nº 625-4, p. 40-47.

Terray E. (1996). Ombres berlinoises. Voyage dans une autre Allemagne. Paris: Odile Jacob, 266 p.

WERLEN B. (1995). Sozialgeographie alltäglicher Regionalisierung, Band 1: Zur Ontologie von Gesellschaft und Raum. Stuttgart: Franz Steiner Verlag, 256 p. 